\title{
Atributos florais em um sistema de polinização especializado: Calathea cylindrica (Roscoe) K. Schum. (Marantaceae) e abelhas Euglossini ${ }^{1}$
}

\author{
ALEX DE ALMEIDA BARRETO² e LEANDRO FREITAS ${ }^{2,3}$
}

(recebido: 10 de agosto de 2006; aceito: 5 de julho de 2007)

\begin{abstract}
Floral traits in a specialized pollination system: Calathea cylindrica (Roscoe) K. Schum. (Marantaceae) and Euglossini bees). Pollination specialization is promoted by floral features that favor pollinator fidelity and hinder the access to resources by other visitors. We investigated the pollination mechanism of one understory herb in southeastern Brazil. Calathea cylindrica flowers year-round and its flowers are peculiar because of the fusion and modification of some elements, asymmetry, secondary pollen presentation, hidden nectar, and a long and narrow tube, which is closed before pollinator visit. Pollination occurs by means of an explosive mechanism, and once it is triggered, the floral parts do not return to their initial position. Thus, there is only a single possibility for pollen transference. Pollinators are female Euglossini bees and all flowers have already been visited by mid-morning. Specific movements with enough force are necessary for the visitors to access the nectar. Bagged flowers secreted around $13 \mu \mathrm{L}$ of nectar with $32 \%$ of sugar concentration. Nectar secretion continues after removals, but in small volumes. Continual flowering, high production of nectar and secretion after pollination may promote pollinator fidelity. The complex floral structure, the secretion in which the flower base (nectar chamber) is dipped, and the narrow tube with inner hairs make the access to nectar difficult and may work as barriers for other visitors. Altogether, these factors seem to regulate the highly specialized pollination system of Calathea cylindrica.
\end{abstract}

Key words - bee, Euglossini, floral specialization, nectar, pollination

RESUMO - (Atributos florais em um sistema de polinização especializado: Calathea cylindrica (Roscoe) K. Schum. (Marantaceae) e abelhas Euglossini). Sistemas de polinização especializados estão ligados a características que incentivam as visitas por polinizadores e desestimulam visitas por outros animais. O mecanismo de polinização de uma espécie de Marantaceae foi estudado com ênfase nessa questão. Calathea cylindrica é uma erva com flores disponíveis o ano todo. As flores são peculiares pela fusão e modificação dos elementos, assimetria, apresentação secundária de pólen, néctar pouco acessível e tubo longo, estreito e fechado até a visita do polinizador. A polinização consiste em um mecanismo explosivo, sendo que uma vez disparado, as estruturas florais não retornam à posição inicial. Assim, há uma única possibilidade para transferência de pólen. Os polinizadores são fêmeas de abelhas Euglossini (Apidae) e no meio da manhã todas as flores já haviam sido visitadas. O acesso ao néctar em flores intactas requer a realização de movimentos específicos e com força apropriada, o que exclui outros insetos. A quantidade de néctar produzido em flores ensacadas foi em torno de $13 \mu \mathrm{L}$ com $32 \%$ de concentração de açúcares. O néctar continua sendo secretado em quantidades pequenas depois do disparo do mecanismo. A floração contínua, a quantidade alta de néctar produzido e a continuidade de secreção após a polinização parecem promover maior fidelidade dos polinizadores. A complexidade estrutural das flores, a secreção que banha externamente a câmara nectarífera e o tubo estreito com pelos internos dificultam o acesso ao néctar e parecem funcionar como barreiras para outros visitantes. Em conjunto, esses fatores parecem ser os determinantes do alto grau de especialização do sistema.

Palavras-chave - abelhas, especialização floral, Euglossini, néctar, polinização

\section{Introdução}

A idéia que a maioria das espécies de angiospermas apresenta sistemas de polinização especializados predominou por muitos anos na literatura específica (e.g., Grant \& Grant 1965, Thompson 1994, Proctor et al. 1996) e, também, em livros didáticos mais gerais (e.g., Raven et al. 2007).

\footnotetext{
1. Parte da monografia apresentada pelo primeiro autor para conclusão da graduação em Ciências Biológicas, Universidade Federal do Estado do Rio de Janeiro (UNIRIO).

2. Jardim Botânico do Rio de Janeiro, Rua Pacheco Leão, 915, 22460-030 Rio de Janeiro, RJ, Brasil.

3. Autor para correspondência: leandro@jbrj.gov.br
}

Isso se deve ao fato que a especialização em certo tipo de polinizador é considerada como um processo central na diversificação das angiospermas (Stebbins 1970, Crepet 1983). Também reflete a influência do conceito de síndromes de polinização (Vogel 1954, Faegri \& van der Pij1 1979) como marco teórico nessa linha de pesquisa, além de certo fascínio exercido pela idéia de coevolução, que frequentemente é exemplificada por casos bem peculiares de interações planta-polinizador, tais como a polinização de espécies de Ficus por agonídeos. A publicação, anos atrás, de alguns artigos bastante influentes que questionavam a validade do conceito de síndromes (Herrera 1996, Waser et al. 1996), gerou um debate 
intenso sobre a prevalência de sistemas generalistas ou especialistas (Johnson \& Steiner 2000, 2003, Gómez 2002, Wilson et al. 2004). Atualmente existe vasta literatura indicando que os sistemas de polinização das angiospermas se distribuem em um contínuo entre os extremos de generalização e especialização (Fenster et al. 2004, Ramírez 2004, Freitas \& Sazima 2006, Waser \& Ollerton 2006) e uma das questões que emerge desse cenário é o entendimento dos fatores que estão ligados ao surgimento e manutenção de sistemas de polinização com alto grau de especialização (Westerkamp 1997, Aigner 2001, 2004, Fenster et al. 2004, Cane \& Sipes 2006).

De acordo com o princípio de Stebbins (1970) do polinizador mais efetivo, uma planta deve especializarse no polinizador mais eficaz e mais abundante quando a disponibilidade deste é confiável. Inversamente, a generalização é favorecida quando a disponibilidade uniforme de um polinizador eficaz é imprevisível ano após ano (Waser et al. 1996). Assim, como modelo geral, a especialização do sistema de polinização é vantajosa para a planta quando acarreta em maior eficiência na polinização e promove fidelidade dos polinizadores, com conseqüente aumento no fluxo de pólen (Johnson \& Steiner 2000). A eficiência na polinização depende de diversos fatores, tais como morfologia floral que favoreça a transferência de pólen entre as flores e por outro lado, desestimule ou impeça o acesso aos recursos florais por outros animais que não os agentes de polinização mais eficientes (Wilson \& Thomson 1996, Aigner 2004), sendo que características relacionadas à eficiência não promovem necessariamente fidelidade dos polinizadores e vice-versa.

A família Marantaceae é predominantemente neotropical e com cerca de 530 espécies, distribuídas em 31 gêneros, constitui elemento característico e conspícuo do sub-bosque de florestas tropicais de baixas altitudes (Endress 1994, Kennedy 2000). Calathea é o maior gênero da família, com aproximadamente 300 espécies (Andersson 1998) e dentre elas, é estimado que ocorram entre 70 e 90 espécies no Brasil (Braga 2005). Apesar da grande representatividade, nenhum estudo sobre a biologia da polinização foi desenvolvido com espécies brasileiras de Calathea. Espécies desse gênero possuem flores com estrutura complexa e mecanismo de polinização peculiar, o que são indicativos de um sistema de polinização com alto grau de especialização, embora esta relação não ocorra em uma espécie de outro gênero de Marantaceae estudada por Locatelli et al. (2004). Os principais polinizadores de espécies de Calathea são abelhas Euglossini (Apidae) (Kennedy 1978, 2000, Endress 1994, Kress \& Beach 1994). Abelhas desse grupo medem de $8 \mathrm{~mm}(70 \mathrm{mg})$ até $28 \mathrm{~mm}$ (113 mg) (Casey et al. 1985) e possuem línguas muito longas, entre 15 e $42 \mathrm{~mm}$ (Roubik \& Hanson 2004), o que permite a exploração de flores com uma ampla gama de comprimento de tubo floral (Borrell 2005). De fato, há registros de Euglossini tomando néctar em espécies de mais de 50 famílias (Roubik \& Hanson 2004). Algumas espécies de Calathea são conhecidas por serem polinizadas por uma única espécie ou por poucas espécies aparentadas de abelhas dos gêneros Eulaema e Euglossa (Kennedy 1978, 2000, Endress 1994). Porém, pouco foi explorado em relação aos atributos que podem estar determinando este alto grau de especialização, em que pese alguns comentários em Kennedy (2000). Assim sendo, este trabalho descreve o mecanismo de polinização de Calathea cylindrica (Roscoe) K. Schum., confirmando sua polinização exclusivamente por espécies de Eulaema e Euglossa e apresenta uma discussão sobre as características relacionadas ao alto grau de especialização de seu sistema de polinização, particularmente a complexidade estrutural das flores e o ritmo de secreção de néctar.

\section{Material e métodos}

Calathea cylindrica é uma espécie endêmica da Mata Atlântica da Bahia a São Paulo, que forma fragmentos clonais (figura 1) com distribuição agregada, preferencialmente em locais com luz direta somente em parte do dia, tais como bordas e clareiras na mata (J.M.A. Braga, comun. pess.). Foram estudados indivíduos que ocorrem espontaneamente no arboreto do Jardim Botânico do Rio de Janeiro, em adjacências de áreas cobertas com Mata Atlântica submontana ( $\left.c a .43^{\circ} 13^{\prime} \mathrm{W}, 22^{\circ} 58^{\prime} \mathrm{S}\right)$. Para o estudo fenológico, 20 fragmentos clonais foram marcados em diferentes áreas do arboreto. Um ramete de cada fragmento foi sorteado e acompanhado mensalmente, de novembro de 2003 a outubro de 2004, para contagem de botões, flores e frutos.

Flores frescas ou fixadas em FAA em etanol $70 \%$ foram observadas sob estereomicroscópio para descrição da morfologia floral. Dados morfométricos foram obtidos com auxílio de paquímetro em 20 flores de diferentes indivíduos, analisadas sob estereomicroscópio. As seguintes medidas foram tomadas: comprimento total (da base do ovário ao ápice), comprimento efetivo do tubo da corola (da base da câmara nectarífera à abertura em "V" do tubo) e diâmetro interno do tubo na altura da abertura e na porção mediana da câmara nectarífera. Para estudo dos nectários em microscopia óptica, foram utilizados ovários de duas flores fixadas em FAA em etanol $70 \%$ e incluídas em historresina. Foram montadas duas séries de lâminas, uma de cortes transversais e outra de longitudinais, com espessura de 9 a $12 \mu \mathrm{m}$ e corados com azul de toluidina. Grãos de pólen retirados de uma flor por inflorescência de 16 fragmentos clonais tiveram a viabilidade estimada pelo teste de carmim acético (Radford et al. 1974). A receptividade do estigma foi verificada pela manhã e à tarde, 
em campo, utilizando-se 60 flores de indivíduos diferentes, através do teste de atividade de catalase, com a aplicação de gotas de peróxido de hidrogênio (Zeisler 1938). Para diminuir possíveis artefatos do método, a concentração de peróxido utilizada foi ajustada através de teste piloto com botões em estágio inicial de desenvolvimento.

O volume e a concentração de açúcares do néctar foram medidos, respectivamente, com auxílio de micro-seringas (Hamilton ${ }^{\circledR}$ ) e refratômetro de bolso (Quimis ${ }^{\circledR}$ ), em flores ensacadas com sacos de "voile" no dia anterior. O valor da concentração de solutos sólidos registrado no refratômetro foi considerado como valor de concentração de açúcares. As medições foram realizadas de duas formas. Foi quantificado o néctar acumulado em flores retiradas das inflorescências no momento da medição em diferentes períodos, pela manhã entre 8 h00 e $10 \mathrm{~h} 00(n=18)$, início da tarde, de $13 \mathrm{~h} 00$ às $14 \mathrm{~h} 30$ $(n=19)$ e no fim da tarde, após às $17 \mathrm{~h} 00(n=6)$. Assim, foi realizada uma única medição com posterior descarte da flor. Essa medida permitiu calcular a produção média de néctar e, consequentemente, determinar o padrão de secreção de néctar. Em outro grupo de flores foram feitas medidas repetidas em cada flor com intervalos de duas horas, mantendo as flores ensacadas entre as medições, de acordo com Galetto \& Bernardello (1992). Esse conjunto de flores foi dividido em cinco grupos com seis flores cada; as medições do néctar do primeiro grupo iniciaram às $7 \mathrm{~h} 00$, do segundo grupo às $9 \mathrm{~h} 00$ e assim sucessivamente até o último grupo, às $15 \mathrm{~h} 00$. As medidas foram tomadas com extremo cuidado, porém existe possibilidade de alguma injúria às flores, especialmente nas últimas medições. Com essas medidas é possível determinar o efeito de remoção na produção de néctar, o qual simula a retirada de néctar pelos polinizadores.

Para verificação do sistema reprodutivo, inflorescências de 13 fragmentos clonais foram ensacadas para impedir visitas às flores. Cada flor foi mantida ensacada depois de efetuada a polinização manual (auto ou cruzada) ou sem qualquer manipulação para teste de autopolinização espontânea ou apomixia. Algumas flores, não ensacadas previamente, foram marcadas e expostas aos polinizadores como controle. A quantidade de frutos formados por flor (i.e., fecundidade) foi utilizada como estimativa do sucesso reprodutivo.

O mecanismo de polinização e os visitantes florais foram estudados diretamente no campo e com auxílio de imagens e com manipulação de flores frescas em laboratório. Para determinação dos polinizadores foram realizadas observações diretas das flores do amanhecer ao entardecer em diferentes épocas do ano. A freqüência de visitas foi quantificada mensalmente em fragmentos clonais escolhidos por sorteio, dentre os fragmentos com flores naquele mês no agrupamento de plantas escolhido para as observações. Esses dados foram coletados através de observações focais no período da manhã, entre novembro de 2003 e outubro de 2004, totalizando 36 horas. O número de flores e o ramete visitado em cada fragmento foram anotados para cada visita. Alguns insetos visitantes foram coletados e montados para identificação posterior.

\section{Resultados}

Biologia floral, sistema reprodutivo e fenologia (figuras 1-8) - C. cylindrica é uma erva com ca. 1,5 m de altura e inflorescências do tipo espiga, pedunculadas, terminais, densas, eretas (figuras 1,2) e com brácteas verdes. As flores abrem sequencialmente da base para o ápice da inflorescência, em mais de um ciclo (Endress 1994 para detalhes). As flores estão dispostas espiraladamente nas inflorescências (figura 2), que possuem uma secreção com pequena viscosidade acumulada entre as brácteas, banhando as flores até a altura da câmara nectarífera. Sobre cada uma das brácteas é apresentada uma sequiência de pares de flores e cada par é protegido por uma bractéola. As flores de cada par abrem concomitantemente ou seqüencialmente. O comprimento total da flor é de 4,18 $\pm 0,12 \mathrm{~cm}(n=20)$. São assimétricas (figuras 3,4$)$, de modo que uma é a imagem invertida da outra, trímeras, tubulosas, claras, com coloração amarelo-esverdeada, sem odor perceptível e duram um dia. O cálice constituise de três sépalas livres (verdes e transparentes). A corola forma um tubo alargado na base (câmara nectarífera) e afilado na porção mediana e apresenta três lobos apicais alargados. Esse alargamento apical abriga as partes livres do gineceu e do androceu (as partes não livres encontram-se fundidas ao tubo da corola) (figura 5). A parede interna da porção mais estreita do tubo encontrase coberta por pelos. O comprimento efetivo do tubo é de 2,08 $\pm 0,15 \mathrm{~cm}(n=20)$, o diâmetro do tubo na altura da abertura é de $0,18 \pm 0,01 \mathrm{~cm}(n=20)$ e o diâmetro da câmara nectarífera é de $0,28 \pm 0,02 \mathrm{~cm}(n=20)$. O ovário é ínfero, de placentação axilar e trilocular, com um único óvulo por lóculo. Os nectários são encontrados nos septos ovarianos (três) e desembocam na luz do tubo da corola em um único ponto. O estilete possui uma projeção perpendicular próxima à sua extremidade, a qual abriga o estigma (côncavo, úmido e não papiloso). O ângulo formado entre essa projeção e a extremidade do estilete (depressão estilar) representa o local de deposição do pólen (i.e., apresentação secundária de pólen) (figura 6). $\mathrm{O}$ androceu é formado por um estame - com uma teca apenas - o qual está unido ao tubo da corola juntamente com três estaminódios (externo, cuculado e caloso) e o estilete (figura 5). Os grãos de pólen são brancos, globosos e encontram-se unidos formando uma massa. O pólen é totalmente depositado na depressão estilar ainda na fase de botão, devido ao alongamento do estilete, que "raspa" o pólen da antera, assim no momento da antese a antera encontra-se vazia.

A viabilidade dos grãos de pólen coletados em torno das 10 h00 foi quase $100 \%$ ( $n=16$ flores). Todos os 

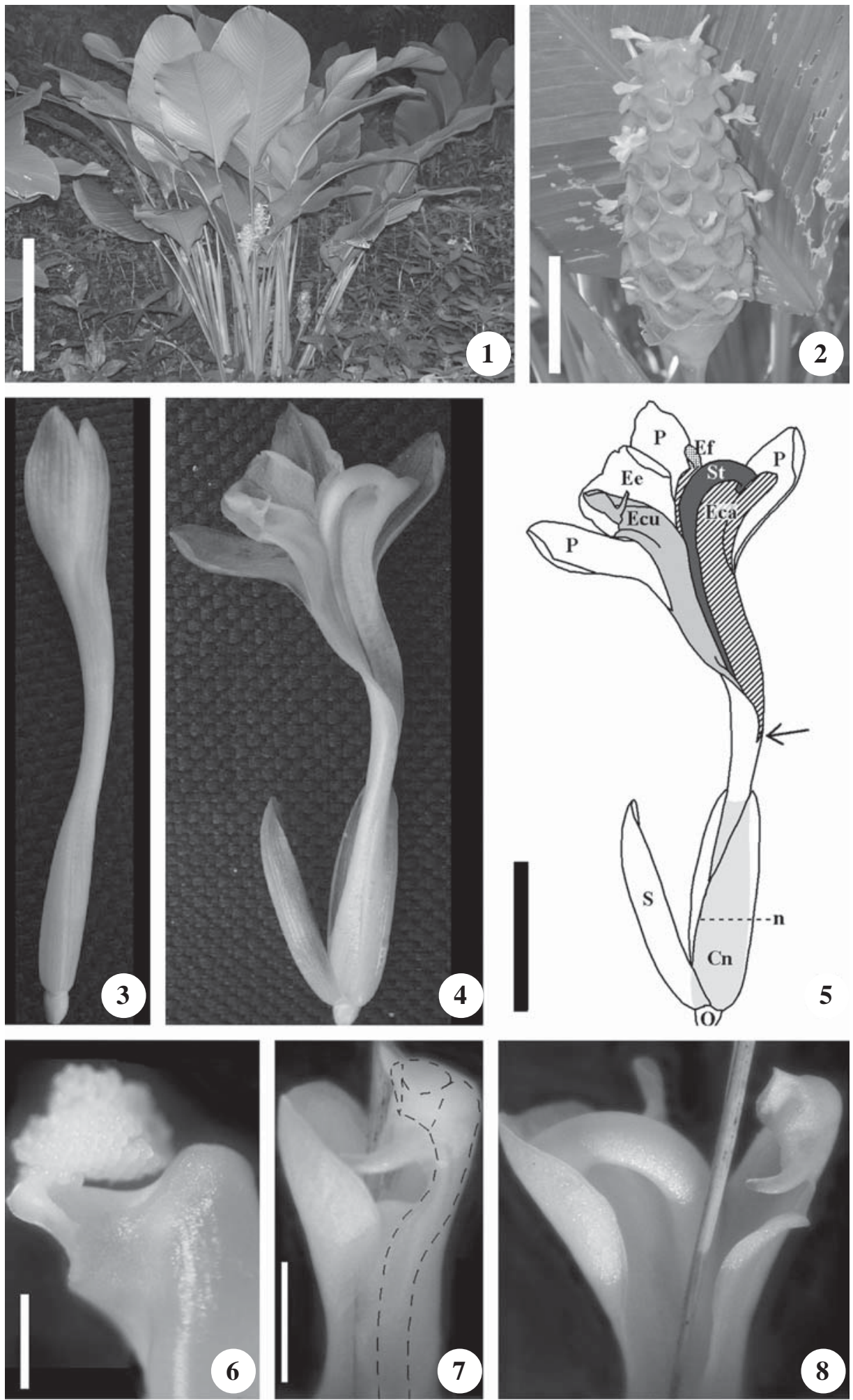

Figuras 1-8. Aspectos morfológicos de Calathea cylindrica. 1. Hábito. 2. Inflorescência. 3. Flor em antese, não visitada. 4. Flor após disparo do mecanismo de polinização. 5. Esquema da flor mostrando as peças (seta = abertura em "V" do tubo da corola; $\mathrm{Cn}$ = câmara nectarífera; Eca = estaminódio caloso; Ecu = estaminódio cuculado; Ee = estaminódio externo; Ef = estame; $\mathrm{n}=$ altura da coluna de néctar em flor recém aberta; $\mathrm{O}=$ ovário; $\mathrm{P}=$ pétalas; $\mathrm{S}=$ sépalas; $\mathrm{St}=$ estilete). 6 . Massa de pólen sobre parte convexa na extremidade apical do estilete. 7-8. Simulação do disparo do mecanismo de polinização (estaminódio externo e as pétalas foram retirados da flor). 7. Posição inicial, o tracejado indica a posição do estilete coberto pelo estaminódio cuculado. 8. Posição após o disparo. Barras = $70 \mathrm{~cm}(1), 5 \mathrm{~cm}(2), 1 \mathrm{~cm}(3,4,5), 1 \mathrm{~mm}(6), 5 \mathrm{~mm}(7,8)$. 
estigmas testados por volta das $8 \mathrm{~h} 00(n=30)$ apresentaram resultado positivo para a estimativa de receptividade, contra $73 \%$ de resultado positivo por volta das $16 \mathrm{~h} 00$ ( $n$ =30). A espécie é auto-compatível, não havendo diferença na produção de frutos entre polinizações cruzadas, auto-cruzamentos e flores expostas aos polinizadores $\left(\chi^{2}=3,17, g l=2, P=0,2043\right)$ (tabela 1). Flores não-manipuladas produziram frutos, indicando a possibilidade de auto-polinização espontânea ou mesmo apomixia (tabela 1), porém em quantidades menores que os outros três tratamentos $\left(\chi^{2}=10,5, g l=3, P=0,0147\right)$.

Tabela 1. Resultados dos experimentos de polinização para determinação do sistema reprodutivo de Calathea cylindrica no Jardim Botânico do Rio de Janeiro, Brasil.

Table 1. Fruit set from hand-pollinations in Calathea cylindrica at Jardim Botânico do Rio do Janeiro, Brazil.

\begin{tabular}{lccc}
\hline Tratamento & $\begin{array}{c}\text { No de } \\
\text { flores }\end{array}$ & $\begin{array}{c}\text { Frutos } \\
\text { formados }\end{array}$ & $\begin{array}{c}\text { Fecundidade } \\
(\% \text { frutos } \\
\left.\text { flores }^{-1}\right)\end{array}$ \\
\hline Autopolinização & 39 & 10 & 25,64 \\
Polinização cruzada & 37 & 13 & 35,14 \\
Polinização natural & 35 & 14 & 40,00 \\
Flores não manipuladas & 35 & 6 & 17,14 \\
\hline
\end{tabular}

Cada inflorescência apresenta flores por alguns meses e uma média de $11 \pm 5,01(n=20)$ flores abrem por dia. Porém, a produção de inflorescências é assincrônica dentro de cada fragmento clonal e entre fragmentos, de modo que ao nível populacional, a floração é contínua, mas com um pico entre outubro e fevereiro (figura 9). $\mathrm{O}$ amadurecimento do fruto ocorre em torno de dois meses após a polinização da flor.

Polinizadores, mecanismo de polinização e secreção de néctar (figuras 10-14) - Duas espécies de abelhas da tribo Euglossini (Apidae) foram observadas polinizando as flores (figuras 10-11), Eulaema cingulata (Fabricius), cuja glossa mede $c a .2,2 \mathrm{~cm}$ e E. nigrita Lepeletier com glossa de $c a \cdot 1,8 \mathrm{~cm}$. Eulaema cingulata foi observada durante todo o ano e sua freqüência média de visitas entre os meses foi de $0,9 \pm 1,07$ visitas por inflorescência por hora $(n=12)$, sendo máxima em agosto de 2004, com mais de 3,14 visitas por hora. As visitas iniciaram ao amanhecer e embora mais freqüientes pela manhã, as abelhas dessa espécie visitaram as flores em busca de néctar sistematicamente ao longo de todo dia. Já E. nigrita foi vista ocasionalmente, apenas na primavera e verão em cinco dos doze meses de estudo, e com frequiência média bem inferior a $E$. cingulata $(0,07 \pm 0,03$ visitas por inflorescência por hora, $n=5)$. Uma espécie não identificada de Lepidoptera foi observada algumas vezes visitando flores já disparadas e aparentemente não pode realizar polinização, uma vez que durante as visitas sua tromba não contata os grãos de pólen depositados na depressão estilar, que se encontra envolta pelo estaminóide caloso (figura 8).

No início da antese, a flor apresenta apenas uma pequena abertura entre as pétalas (figura 3 ) e permanece deste modo até murchar, a menos que seja visitada por algum animal capaz de abri-la para acessar o néctar. A polinização consiste num mecanismo explosivo e envolve o estilete e os estaminódios. O estaminódio cuculado, que tem posição oposta ao caloso, constitui uma espécie de capuz que encobre e mantém pressionado o estilete. Nesse estaminódio é encontrada uma projeção digitiforme em sua lateral mais externa, que constitui o "gatilho" desse mecanismo (figuras 5, 7). Antes do disparo, os estaminódios cuculado e caloso formam uma "calha" e o gatilho encontra-se posicionado entre eles. Além disso, a flor possui uma curvatura para baixo na altura da abertura em "V". Ao buscar néctar, a abelha agarra a flor com as pernas, introduz a língua na extremidade da flor e, conforme ela desliza pelo centro da "calha" e se desloca de baixo para cima para fazer a curva, empurra o "gatilho" para cima e para a lateral libertando o estilete, que salta para o lado oposto da flor indo ao encontro do estaminódio caloso (figuras 7-8, 12-14). É nesse caminho que o estilete atinge a abelha e deposita a massa de pólen na glossa, na porção mais próxima à cabeça (figura 13). Nessa ocasião ocorre a total dispersão de pólen. Uma vez disparado o mecanismo, as estruturas florais não

Figures 1-8. Morphological aspects of Calathea cylindrica. 1. Habit. 2. Inflorescence. 3. Unvisited flower in anthesis. 4. Flower after the pollination mechanism was triggered. 5. Schematic drawing of the flower showing elements (arrow = "V" opening of the corolla tube; $\mathrm{Cn}=$ nectar chamber; Eca = callous staminode; $\mathrm{Ecu}=$ cucullate staminode; $\mathrm{Ee}=$ outer staminode; Ef = stamen; $\mathrm{n}=$ mean level of nectar column in an unvisited flower; $\mathrm{O}=$ ovary; $\mathrm{P}=$ petals; $\mathrm{S}=$ sepals; $\mathrm{St}=$ style). 6 . Pollen mass on the convex side of the style top. 7-8. Simulation of the pollination mechanism triggering (the petals and the outer staminode were removed). 7. Initial position, dashed figure indicates the style covered by the cucullate staminode. 8. Position after triggering. Bars $=70 \mathrm{~cm}(1), 5 \mathrm{~cm}(2), 1 \mathrm{~cm}(3,4,5), 1 \mathrm{~mm}(6), 5 \mathrm{~mm}(7,8)$. 


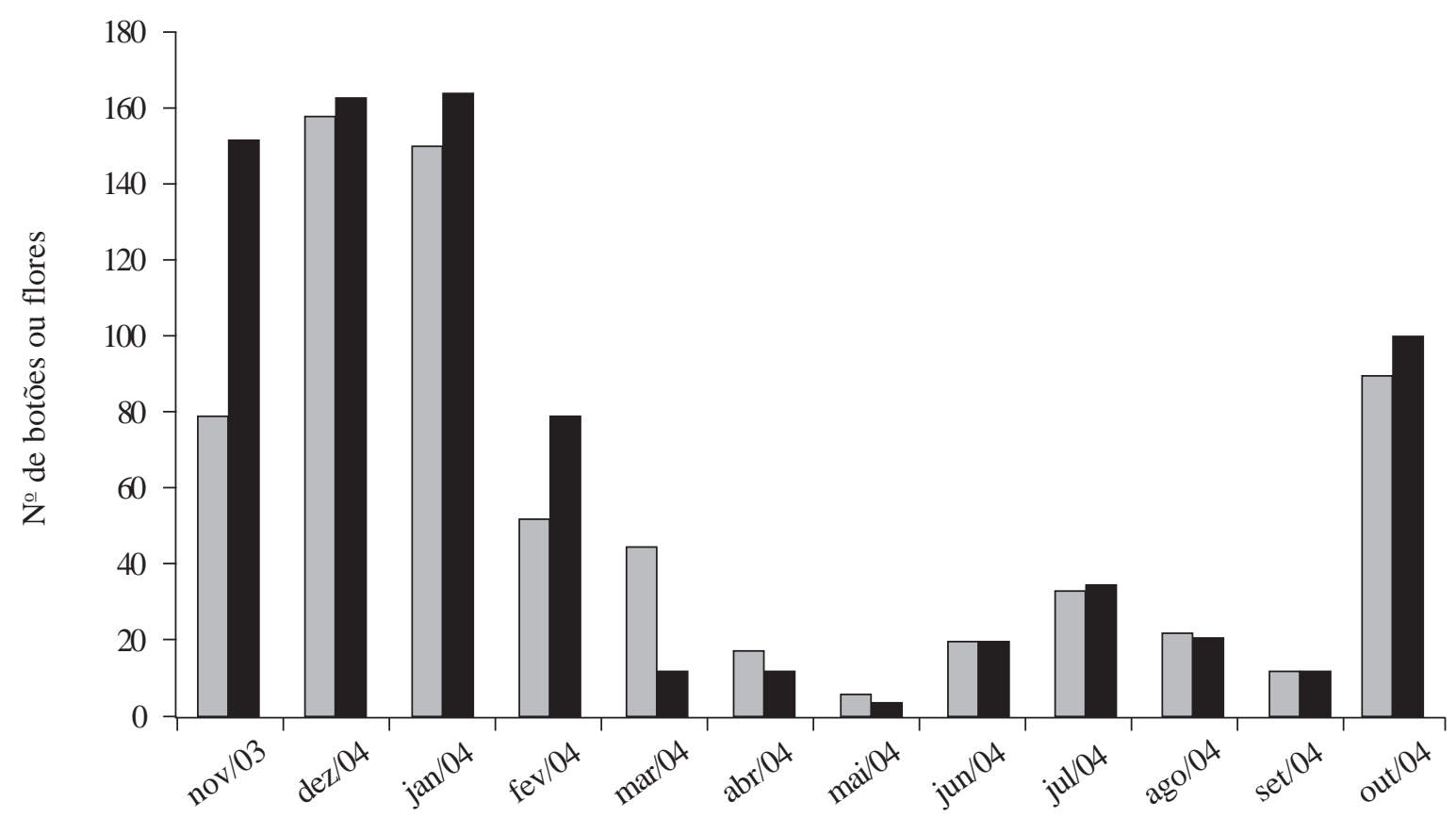

Figura 9. Floração de Calathea cylindrica no Jardim Botânico do Rio de Janeiro. Dados representam o número acumulado de botões e flores de 20 inflorescências ( $\square=$ botões; $\square=$ flores).

Figure 9. Flowering phenology of Calathea cylindrica at Jardim Botânico do Rio de Janeiro, Brazil. Total number of buds and flowers of 20 inflorescences ( $\square=$ buds; $\square=$ flowers).

retornam à posição inicial, de modo que há uma única possibilidade para transferência de pólen.

A quantidade média de néctar pela manhã em flores ensacadas foi de $12,7 \pm 5,13 \mu \mathrm{L}$ com concentração de açúcares de $32,3 \pm 7,41 \%(n=18)$. A secreção de néctar ocorre antes da abertura da flor e cessa caso não haja retirada, portanto, as quantidades encontradas em flores mantidas ensacadas são equivalentes entre os períodos da manhã e início da tarde $(12,3 \pm 5,36 \mu \mathrm{L}, 32,6 \pm 8,31 \%$, $n=19)$ ou final da tarde $(13,5 \pm 0,89 \mu \mathrm{L}, 37,9 \pm 4,27 \%$, $n=6)$. A importância desses resultados para entendimento do sistema é restrita, uma vez que por volta das 9h00, todas as flores de $C$. cylindrica já haviam sido visitadas pelos polinizadores. Em relação aos efeitos de remoção de néctar, a secreção prossegue depois de retiradas, porém em quantidades pequenas (tabela 2).

\section{Discussão}

As flores de Calathea cylindrica apresentam várias características morfológicas peculiares, tais como fusão e modificação de elementos, zigomorfia, tubo longo, extremamente estreito e praticamente fechado até a visita do polinizador, apresentação secundária de pólen, néctar pouco acessível e movimento explosivo e irreversível do estilete durante a polinização. Essas características são comuns às flores de muitas espécies do gênero e da família como um todo (Kennedy 1978, 2000). Como consequência dessas características florais, o disparo do mecanismo de polinização requer a realização de movimentos específicos e com força apropriada pelo polinizador, o que provavelmente exclui insetos menores ou incapazes de realizar os movimentos corretos. Neste sentido, a complexidade estrutural das flores parece ser um fator determinante da polinização de $C$. cylindrica exclusivamente por fêmeas de Euglossini em busca de néctar. A polinização por abelhas desse grupo, observada para essa espécie de Calathea em uma área do sudeste do Brasil, coincide com os registros de polinizadores e com o conseqüente alto grau de especialização do sistema de polinização de outras espécies do gênero com flores semelhantes na América Central (Kennedy 1978, 2000, Ackerman 1985, Kress \& Beach 1994).

A quantidade de néctar secretada por $C$. cylindrica (ca. $5 \mathrm{mg}$ de açúcar por dia) se encaixa na classe de valores típica de flores polinizadas por abelhas Euglossini (e.g., Roubik et al. 1995), as quais atingem altas temperaturas corporais quando em atividade e por isso precisam 
Tabela 2. Efeito de remoção de néctar em Calathea cylindrica. Valores são médias $\pm s ; n$ representa o número de flores.

Table 2. Nectar removal effect in Calathea cylindrica. Values are mean $\pm s ; n$ means flower number.

\begin{tabular}{|c|c|c|c|c|c|c|c|}
\hline \multicolumn{8}{|c|}{ Volume de néctar $(\mu \mathrm{L})$} \\
\hline Set & $n$ & $7 \mathrm{~h} 00$ & $9 \mathrm{~h} 00$ & $11 \mathrm{~h} 00$ & $13 \mathrm{~h} 00$ & $15 \mathrm{~h} 00$ & Total \\
\hline 1 & 6 & $6,75 \pm 1,99$ & $3,25 \pm 6,09$ & $1,58 \pm 0,97$ & 0 & 0 & $11,6 \pm 5,67$ \\
\hline 2 & 6 & & $9,83 \pm 4,99$ & $0,17 \pm 0,26$ & 0 & 0 & $10,0 \pm 5,07$ \\
\hline 3 & 6 & & & $10,25 \pm 4,70$ & $1,92 \pm 2,42$ & 0 & $12,2 \pm 4,95$ \\
\hline 4 & 6 & & & & $5,92 \pm 3,12$ & $1,58 \pm 2,29$ & $7,5 \pm 4,36$ \\
\hline 5 & 6 & & & & & $7,42 \pm 3,57$ & $7,4 \pm 3,57$ \\
\hline \multicolumn{8}{|c|}{ Concentração de néctar $\left(\% \mathrm{p} \mathrm{p}\right.$ de açúcares $\left.{ }^{-1}\right)$} \\
\hline Set & $n$ & $7 \mathrm{~h} 00$ & $9 \mathrm{~h} 00$ & $11 \mathrm{~h} 00$ & $13 \mathrm{~h} 00$ & $15 \mathrm{~h} 00$ & Média \\
\hline 1 & 6 & $33,08 \pm 3,77$ & $7,17 \pm 8,01$ & $8,80 \pm 11,03$ & 0 & 0 & $16,8 \pm 14,46$ \\
\hline 2 & 6 & & $23,50 \pm 16,77$ & 0 & 0 & 0 & $23,5 \pm 16,77$ \\
\hline 3 & 6 & & & $25,00 \pm 16,43$ & $14,50 \pm 16,92$ & 0 & $20,8 \pm 16,58$ \\
\hline 4 & 6 & & & & $27,75 \pm 10,80$ & $10,80 \pm 14,94$ & $20,0 \pm 15,03$ \\
\hline 5 & 6 & & & & & $36,50 \pm 1,97$ & $36,5 \pm 1,97$ \\
\hline
\end{tabular}

consumir grandes quantidades de néctar (Dressler 1982). Como exemplo, Euglossa imperialis necessita de $40 \mathrm{mg}$ de açúcar por dia para manter sua massa corpórea (Borrell 2005). As flores de néctar polinizadas por abelhas Euglossini formam uma síndrome de polinização bem definida, denominada euglossofilia (Ackerman 1985, Kay \& Schemske 2003), que inclui flores com coloração clara, tubos longos e néctar de difícil acesso produzido em grandes quantidades em comparação a flores polinizadas por outros grupos de abelhas. As flores de C. cylindrica, considerando sua morfologia e produção de néctar, se encaixam nesta síndrome. Além disso, floração longa com baixa amplitude (i.e., poucas flores por dia) também são características tipicamente relacionadas com polinização por Euglossini e outras abelhas que forrageiam em linhas de captura ("traplining") (Janzen 1971, Gentry 1974, Dressler 1982, Kennedy 2000). Tem sido argumentado que a presença de poucas flores por dia e o comportamento de forrageio em linhas de captura favorece o fluxo de pólen, na medida em que conduz o polinizador a visitar um grande número de indivíduos diferentes (Janzen 1971), porém, se estes polinizadores apresentam comportamento generalista visitando flores de diversas espécies em cada ronda de visitas, o fluxo de pólen pode ficar restrito aos indivíduos mais próximos.

Em relação à morfometria, existe uma coincidência entre os comprimentos do tubo da corola e da glossa das duas espécies de abelhas polinizadoras, sendo que no caso de Eulaema nigrita, que possui a glossa um pouco mais curta que o tubo, também existe correspondência morfológica, pois a pequena espessura da parede do tubo e a sua abertura em "V" funcionam como um picote, que reduz o comprimento efetivo do tubo durante a visita, encurtando a distância ao néctar. Encaixes morfométricos, tal como o descrito acima, podem suportar especulações acerca de restrições de largura de nicho trófico para ambos os lados da interação, i.e., pequeno número de polinizadores, tal qual observado para $C$. cylindrica e, do outro lado, pequeno número de espécies de plantas polinizadas pelas espécies de abelhas. Aparentemente, tal modelo com interações planta-polinizador simétricas não é o caso para Euglossini, uma vez que geralmente forrageiam em muitas flores entre aquelas que podem fisicamente extrair néctar, incluindo flores com tubo bem mais curto que o comprimento de sua glossa (Borrell 2005).

A progressiva especialização dos sistemas de polinização pode se dar através de dois mecanismos gerais: a evolução de características florais que tornam as flores mais adequadas aos seus polinizadores principais (maior eficiência na polinização - sensu Stebbins 1970) e através de caracteres que impossibilitem ou dificultem que outros visitantes florais (pilhadores ou polinizadores pouco eficientes) tenham acesso a seus recursos (Wilson \& Thomson 1996, Johnson \& Steiner 2000, Aigner 2004). A fenologia e as características morfológicas e do néctar das flores de $C$. cylindrica discutidas acima parecem promover maior eficiência de abelhas Euglossini na polinização, uma vez que favorecem o transporte de pólen 

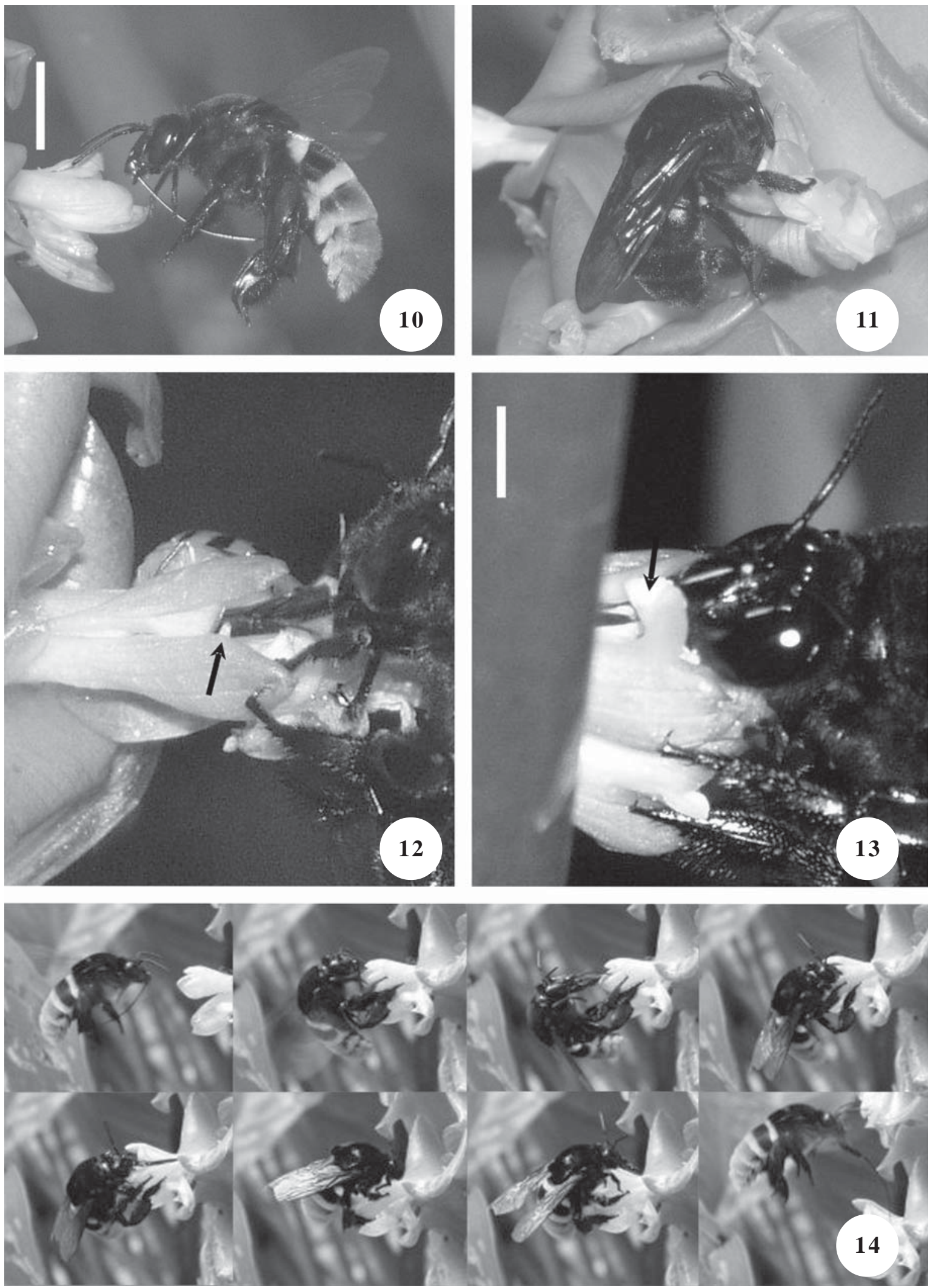

Figuras 10-14. Polinizadores de Calathea cylindrica. 10. Eulaema cingulata se aproximando da flor. 11. Eulaema nigrita tomando néctar. 12-13. Disparo do mecanismo de polinização por E. cingulata. 12. Flor em posição inicial, com estilete coberto pelo estaminódio cuculado (seta = língua da abelha tocando o gatilho do mecanismo). 13. Momento de disparo do mecanismo $($ seta $=$ massa de pólen sobre o estilete). 14. Sequência de visita a uma flor por $E$. cingulata. Barras $=5 \mathrm{~mm}(10,11), 2,5 \mathrm{~mm}(12,13)$. 
entre plantas e o contato da abelha primeiro com o estigma e minimizam a perda de pólen ao depositá-lo em um ponto específico na glossa e promover a constância dos polinizadores. Por outro lado, a copiosa secreção viscosa que banha externamente a câmara nectarífera e o tubo estreito e com presença de pelos na parede interna parecem funcionar como barreiras que desestimulam outros visitantes. De fato, apenas uma espécie de lepidóptero foi observada como pilhador de néctar. $\mathrm{O}$ fato do néctar nessa espécie ser de difícil acesso pode ser um fator que favorece a fidelidade dos Euglossini, pois reduziria drasticamente a competição inter-específica por seu néctar (Feinsinger 1987, Johnson et al. 2004). Além disso, alguns modelos, que consideram o custo de aprendizagem para manipulação das flores, predizem que a fidelidade de abelhas será maior em flores complexas com maiores custos de manipulação para obtenção do néctar (Chittka et al. 1999). Ao menos outros dois fatores em C. cylindrica podem ser interpretados como positivos para a promoção de fidelidade por essas abelhas, caracterizadas por terem vida longa e demanda energética alta (Roubik 1989). São eles, a floração longa em cada indivíduo com baixa sincronia entre indivíduos - o que proporciona oferta de flores o ano todo - e a continuidade de secreção de néctar, mesmo que esta seja em quantidade pequena, após a primeira visita do polinizador e o conseqüiente disparo do mecanismo de polinização.

Outras espécies são conhecidas por secretar néctar após a polinização (Aizen \& Basilio 1998, Koopowitz \& Marchant 1998, Stpiczynska 2003). Esse fenômeno é atribuído ao fato de polinizações múltiplas em uma flor aumentarem a eficiência de fertilizações e a produção de sementes, bem como a exportação de pólen (e.g., Rathcke 1992, Neiland \& Wilcock 1995). Porém, essa idéia não faz sentido para $C$. cylindrica, na qual o disparo do mecanismo de polinização é oportunidade única para exportar e para receber pólen. Neste sentido, a continuidade de produção de néctar após o disparo representaria desperdício energético, aparte explicações não-adaptativas, que consideram o custo para produção de néctar baixo (e.g., Aizen \& Basilio 1998). Uma hipótese para esse sistema é que a produção extra de néctar pode desestimular as abelhas a procurarem outras fontes de recurso, o que promoveria aumento de fidelidade pelos polinizadores. Tal comportamento tem sido considerado vantajoso para a planta por reduzir a competição interespecífica pelos serviços dos polinizadores (Locatelli et al. 2004), favorecendo a transferência de pólen entre co-específicos (Chittka et al. 1999 e Johnson et al. 2004). Uma explanação alternativa para a secreção de néctar após a polinização da flor é que esta seria resultado de competição entre os indivíduos de C. cylindrica (i.e., intraespecífica) pelos polinizadores, pois aumentaria a chance das abelhas manterem em sua rota de forrageamento na manhã seguinte aquele indivíduo visitado à tarde. Essa idéia tem como base que abelhas em geral (Waddington 1983) e particularmente as da tribo Euglossini (Janzen 1971, Ackerman et al. 1982) possuem grande capacidade de aprendizado e de memória de fontes de recurso. Hipótese semelhante foi previamente sugerida para explicar a evolução de tubos longos em flores polinizadas por Euglossini (Rathcke 1992, Kay \& Schemske 2003) e por beija-flores (Garrison \& Gass 1999). Apesar disto, os dados disponíveis para a interação entre espécies de Calathea e Euglossini não permitem conclusões sobre prevalência de competição intra- ou inter-específica na seleção dos caracteres deste sistema. Em suma, o alto grau de especialização do sistema de polinização de $C$. cylindrica parece ser determinado pela complexidade estrutural das flores e também pelo padrão de floração, quantidade de néctar e continuidade de secreção de néctar após ocorrência de polinização, que, em conjunto, promovem maior fidelidade das abelhas.

Associações entre espécies de plantas e interações planta-animal formam redes no espaço, de tal forma que a perda de uma espécie pode causar uma reação em cadeia resultando na extinção de outras espécies, no mesmo ou em diferentes habitats (Gilbert 1980). Assim, plantas que dependem de uma espécie ou de um grupo restrito de polinizadores, em tese, correm risco mais alto de serem extintas. Porém, certas características de plantas dependentes de poucos polinizadores, como propagação vegetativa, ciclo de vida longo, autopolinização facultativa, alta variação fenotípica entre indivíduos, baixa sincronia de desenvolvimento, uso de diferentes tipos de habitats e poligamia podem constituir mecanismos compensatórios, que minimizam o risco de extinção e permitem a existência de uma população por centenas de anos sem seus parceiros mutualísticos (Bond 1994, Vazquez \& Simberloff 2002). Apesar da alta especificidade do sistema de polinização

Figures 10-14. Pollinators of Calathea cylindrica. 10. Eulaema cingulata approaching a flower. 11. Eulaema nigrita taking nectar. 12-13. Pollination mechanism triggering by E. cingulata. 12. Flower in initial position with the style covered by the cucullate staminode (arrow = bee tongue touching the trigger). 13. Mechanism triggering (arrow = pollen mass on the style). 14. Visit of sequence to a flower by E. cingulata . Bars $=5 \mathrm{~mm}(10,11), 2.5 \mathrm{~mm}(12,13)$. 
de C. cylindrica, não existe necessariamente alta suscetibilidade desta espécie a mudanças populacionais de seus polinizadores, já que é uma planta autocompatível, com algum grau de autopolinização espontânea ou apomixia, além de apresentar propagação vegetativa. Dessa forma, sua população poderia ser mantida em um cenário de perda das abelhas polinizadoras, apesar do possível aumento de endogamia.

Agradecimentos - A João Marcelo A. Braga, pelas informações e discussões sobre Calathea; André S. Zaú, Cristiana Koschnitzke e Rosani C. Arruda, pelas sugestões em uma versão prévia; Leila Morgado pela confirmação da identificação das abelhas; Elaine Z. de Souza, Felipe Barreto, Karen G. de Toni e Sandra Barreto, pelo auxílio na obtenção de dados. O primeiro autor foi bolsista PIBIC-CNPq; Faperj (Auxílio instalação) e Petrobras (Programa Mata Atlântica-JBRJ) forneceram apoio financeiro ao projeto.

\section{Referências bibliográficas}

ACKERMAN, J.D. 1985. Euglossine bees and their nectar hosts. In The botany and natural history of Panama (W.G. D'Arcy \& M.D. Correa, eds.). Missouri Botanical Garden, St Louis, p.225-233.

ACKERMAN, J.D., MESLER, M.R., LU, K.L. \& MONTALVO, A.M. 1982. Food-foraging behavior of male Euglossini (Hymenoptera: Apidae): vagabonds or trapliners? Biotropica 14:241-248.

AIGNER, P.A. 2001. Optimality modeling and fitness tradeoffs: when should plants become pollinator specialists? Oikos 95:177-184.

AIGNER, P.A. 2004. Floral specialization without trade-offs: optimal corolla flare in contrasting pollination environment. Ecology 85:2560-2569.

AIZEN, M. \& BASILIO, A. 1998. Sex differential nectar secretion in protandrous Alstroemeria aurea (Alstroemeriaceae): is production altered by pollen removal and receipt? American Journal of Botany 85:245-252.

ANDERSSON, L. 1998. Marantaceae. In The families and genera of vascular plants - Monocotyledons: Alismatanae and Commelinanae (except Gramineae) (K. Kubitzki, ed.) Springer-Verlag, Berlin, v.4, p.278-293.

BOND, W.J. 1994. Do mutualisms matter? Assessing the impact of pollinator and disperser disruption on plant extinction. Philosophical Transactions of the Royal Society of London, Series B 344:83-90.

BORRELL, B.J. 2005. Long tongues and loose niches: evolution of euglossine bees and their nectar flowers. Biotropica 37:664-669.

BRAGA, J.M.A. 2005. Marantaceae: novidades taxonômicas e nomenclaturais III: tipificações, sinonímias e uma nova combinação em Calathea. Acta Botanica Brasilica 19: 763-768.
CANE, J.H. \& SIPES, S. 2006. Characterizing floral specialization by bees: analytical methods and a revised lexicon for oligolecty. In Plant-pollinator interactions: from specialization to generalization (N.M. Waser \& J. Ollerton, eds.). University of Chicago Press, Chicago, p.99-122

CASEY, T.M., MAY, M.L. \& MORGAN, K.R. 1985. Flight energetics of euglossine bees in relation to morphology and wing stroke frequency. Journal of Experimental Biology 116:271-289.

CHITTKA, L., THOMSON, J.D. \& WASER, N.M. 1999. Flower constancy, insect psychology, and plant evolution. Naturwissenschaften 86:361-377.

CREPET, W.L. 1983. The role of insect pollination in the evolution of angiosperms. In Pollination biology (L. Real, ed.). Academic Press, Orlando, p.29-50.

DRESSLER, R.L. 1982. Biology of the orchid bees (Euglossini). Annual Review of Ecology and Systematics 13:373-394.

ENDRESS, P.K. 1994. Diversity and evolutionary biology of tropical flowers. Cambridge University Press, Cambridge.

FAEGRI, K. \& VAN DER PIJL, L. 1979. The principles of pollination ecology. Pergamon Press, $3^{\text {rd }}$ ed., Oxford.

FEINSINGER, P. 1987. Effects of plant species on each other's pollination: is community structure influenced? Trends in Ecology and Evolution 2:123-126.

FENSTER, C.B., ARMBRUSTER, W.S., WILSON, P., DUDASH, M.R. \& THOMSON, J.D. 2004. Pollination syndromes and floral specialization. Annual Review of Ecology, Evolution and Systematics 35:375-403.

FREITAS, L. \& SAZIMA, M. 2006. Pollination biology in a tropical high-altitude grassland in Brazil: interactions at the community level. Annals of the Missouri Botanical Garden 93:465-516.

GALETTO, L. \& BERNARDELLO, L. 1992. Nectar secretion pattern and removal effects in six Argentinean Pitcairnioideae (Bromeliaceae). Botanica Acta 105:292-299.

GARRISON, J.S.E. \& GASS, C.L. 1999. Response of a traplining hummingbird to changes in nectar availability. Behavioral Ecology 10:714-725.

GENTRY, A.H. 1974. Flowering phenology and diversity in tropical Bignoniaceae. Biotropica 6:64-68.

GILBERT, L.E. 1980. Food web organization and the conservation of neotropical diversity. In Conservation biology (M.E. Soule \& B.A. Wilcox, eds.). Sinauer Associates, Sunderland, p.11-34.

GÓMEZ, J.M. 2002. Generalización en las interacciones entre plantas y polinizadores. Revista Chilena de Historia Natural 75:105-116.

GRANT, K. \& GRANT, V. 1965. Flower pollination in the phlox family. Columbia University Press, New York.

HERRERA, C.M. 1996. Floral traits and plant adaptation to insect pollinators: a devil's advocate approach. In Floral biology: studies on floral evolution in animal-pollinated plants (D.G. Lloyd \& S.C.H. Barrett, eds.). Chapman \& Hall, New York, p.65-87.

JANZEN, D.H. 1971. Euglossine bees as long distance pollinators of tropical plants. Science 171:203-205. 
JOHNSON, S.D. \& STEINER, K.E. 2000. Generalization versus specialization in plant pollination systems. Trends in Ecology and Evolution 15:140-143.

JOHNSON, S.D. \& STEINER, K.E. 2003. Specialized pollination systems in southern Africa. South Africa Journal of Science 99:345-348.

JOHNSON, S.D., PETER, C.I. \& ÅGREN, J. 2004. The effects of nectar addition on pollen removal and geitonogamy in the non-rewarding orchid Anacamptis morio. Proceedings of the Royal Society of London, Series B 271:803-809.

KAY, K.M. \& SCHEMSKE, D.W. 2003. Pollinator assemblages and visitation rates for 11 species of neotropical Costus (Costaceae). Biotropica 35:198-207.

KENNEDY, H. 1978. Systematics and pollination of the "closedflowered" species of Calathea (Marantaceae). University of California Publications in Botany, vol. 71, Berkeley.

KENNEDY, H. 2000. Diversification in pollination mechanisms in the Marantaceae. In Monocots: Systematics and Evolution (K.L. Wilson \& D.A. Morrison, eds.). CSIRO Publishing, Collingwood, p.335-344.

KOOPOWITZ, H. \& MARCHANT T.A. 1998. Postpollination nectar reabsorption in the African epiphyte Aerangis verdickii (Orchidaceae). American Journal of Botany 85:508-512.

KRESS, W.J. \& BEACH, J.H. 1994. Flowering plant reproductive systems. In La Selva: Ecology and Natural History of a Lowland Tropical Rainforest (L.A. McDade, K.S. Bawa, G.S. Hartshorn, \& H.A. Hespenheide, eds.). University of Chicago Press, Chicago. p.161-182.

LOCATELLI, E., MACHADO, I.C. \& MEDEIROS, P. 2004. Saranthe klotzschiana (Koer.) Eichl. (Marantaceae) e seu mecanismo explosivo de polinização. Revista Brasileira de Botânica 27:757-765.

NEILAND, M.R.M. \& WILCOCK, C.C. 1995. Maximisation of reproductive success by European Orchidaceae under conditions of infrequent pollination. Protoplasma 187:39-48.

PROCTOR, M., YEO, P. \& LACK, A. 1996. The natural history of pollination. Timber Press, Portland.

RADFORD, A.E., DICKINSON, W.C., MASSEY, J.R. \&, BELL, C.R. 1974. Vascular plant systematics. Harper \& Row Publishers, New York.

RAMÍREZ, N. 2004. Pollination specialization and time of pollination on a tropical Venezuelan plain: variations in time and space. Botanical Journal of the Linnean Society 145:1-16.

RATHCKE, B.J. 1992. Nectar distribution, pollinator behavior, and plants reproductive success. In Effects of resource distribution on animal-plant interactions (M.D. Hunter, T. Ohgushi \& P. N. Price, eds.). Academic Press, San Diego, p.113-138
RAVEN, P.H., EVERT, R.F. \& EICHHORN, S.E. 2007. Biologia vegetal. 7 ed. Guanabara Koogan, Rio de Janeiro.

ROUBIK, D.W. 1989. Ecology and natural history of tropical bees. Cambridge University Press, Cambridge.

ROUBIK, D.W. \& HANSON, P.E. 2004. Abejas de orquídeas de la América tropical: biología y guía de campo. Instituto Nacional de Biodiversidad, INBio, San José, Costa Rica.

ROUBIK, D.W., YANEGA, D., ALUJA, M., BUCHMANN, S.L. \& INOUYE, D.W. 1995. On optimal nectar foraging by some tropical bees (Hymenoptera, Apidae). Apidologie 26:197-211.

STEBBINS, G.L. 1970. Adaptive radiation of reproductive characteristics in angiosperms. I: Pollination mechanisms. Annual Review of Ecology and Systematics 1:307-326.

STPICZYNSKA, M. 2003. Floral longevity and nectar secretion of Platanthera chlorantha (Custer) Rchb. (Orchidaceae). Annals of Botany 92:191-197.

THOMPSON, J.N. 1994. The coevolutionary process. University of Chicago Press, Chicago.

VAZQUEZ, D.P. \& SIMBERLOFF, D. 2002. Ecological specialization and susceptibility to disturbance: conjectures and refutations. American Naturalist 159: 606-623.

VOGEL, S. 1954. Blütenbiologische Typen als Elemente der Sippengliederung, dargestellt anhand der Flora Südafrikas. Botanische Studien 1:1-338.

WADDINGTON, K.D. 1983. Foraging behavior of pollinators. In Pollination biology (L. Real, ed.). Academic Press, New York, p.213-241.

WASER, N.M., CHITTKA, L., PRICE, M.V., WILLIAMS, N.M. \& OLLERTON, J. 1996. Generalization in pollination systems, and why it matters. Ecology 77:1043-1060.

WASER, N.M. \& OLLERTON, J. (eds.) 2006. Plant-pollinator interactions: from specialization to generalization. University of Chicago Press, Chicago.

WESTERKAMP, C. 1997. Flowers and bees are competitors not partners: towards a new understanding of complexity in specialized bee flowers. Acta Horticulturae 437:71-74.

WILSON, P. \& THOMSON, J.D. 1996. How do flowers diverge? In Floral biology: studies on floral evolution in animal-pollinated plants (D.G. Lloyd \& S.C.H. Barrett, eds.). Chapman \& Hall, New York, p.88-111.

WILSON, P., CASTELLANOS, C., HOGUE, J.N., THOMSON, J.D. \& ARMBRUSTER, W.S. 2004. A multivariate search for pollination syndromes among penstemons. Oikos 104:345-361.

ZEISLER, M. 1938. Über die Abgrenzung der eigentlichen Narbenfläche mit Hilfe von Reaktionen. Beihefte zum Botanischen Centralblatt 58:308-318. 